\title{
>
}

(4)

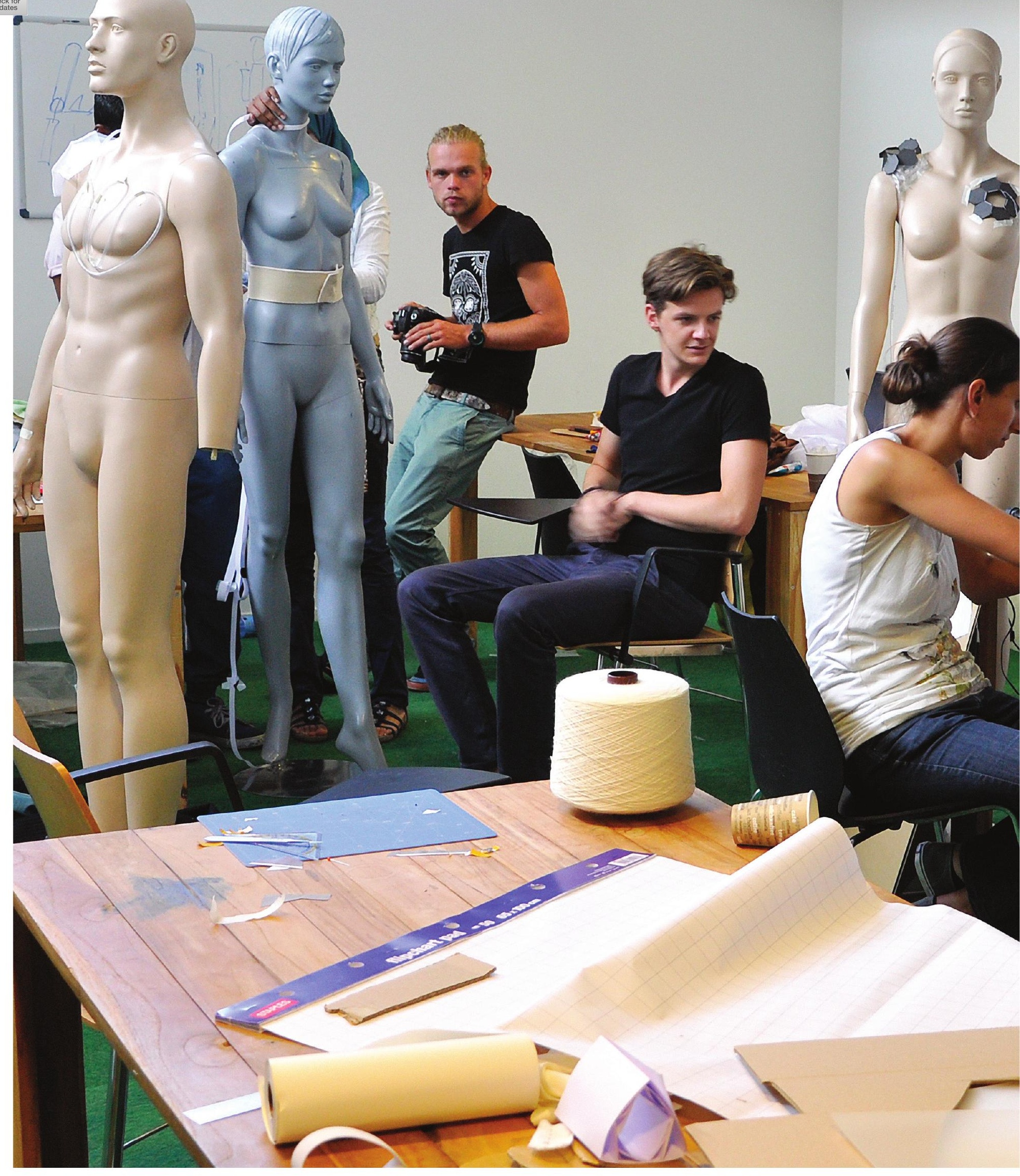




\section{Wearable Senses, Department of Industrial Design, TU Eindhoven}

As told by Oscar Tomico, Stephan Wensveen, Kristi Kuusk, Martijn ten Bhömer, René Ahn, Marina Toeters, and Maarten Versteeg

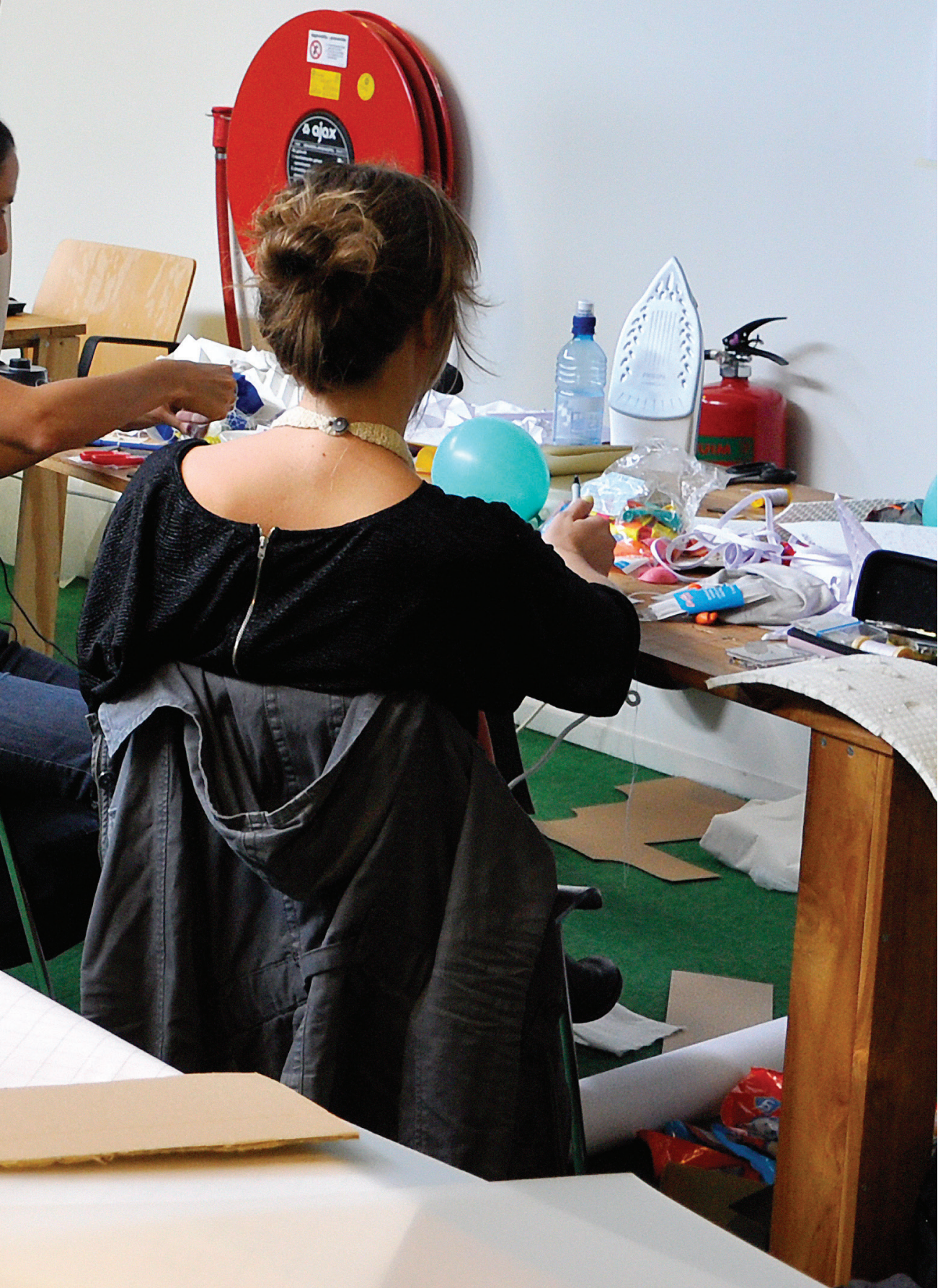

H ow do you describe your lab to visitors? Wearable Senses (WS) focuses on designing close-tothe-body interactions, specifically designs that incorporate wearable computing or smart textiles. It is a community that feels like an emerging multidisciplinary culture, where practitioners from research, education, and industry help and challenge each other on a continual basis.

What is a unique feature of your lab? Wearable Senses aims to integrate research, education, and innovation. Students work in close collaboration with the WS staff and are encouraged to explore design opportunities hands on, which is visible in the open space where students, staff, and coaches work together. However, the focus on intelligent products and systems distinguishes the approach of WS from, for example, textile and fashion schools that offer courses on smart textiles. In line with our educational principles, we advocate a competencycentered and research-through-design approach. This approach can be seen as an iterative transaction between design and research in which skills, knowledge, and attitudes are generated through cycles of designing, building, and experimentally testing experiential prototypes in real-life settings.

This approach is supported by the availability of the tools and materials in our TexLab. Our students and staff not only have the opportunity to work with a variety of textile techniques, such as sewing, knitting, and weaving, but also can use soldering stations to directly integrate electronics into textiles. Further, a materials library provides high-end innovative textile and electronics materials

How many people are in the lab, and what is the mix of backgrounds and roles? At WS, people from very different disciplines work closely together. Interaction and fashion designers, people familiar with the details of human physiology, psychologists, sociologists, and engineers are all required to create propositions that are accepted by end users in the market. This combination, as we learned through experience, is by no means trivial. 


\section{$>{ }_{\text {sersin }}$}
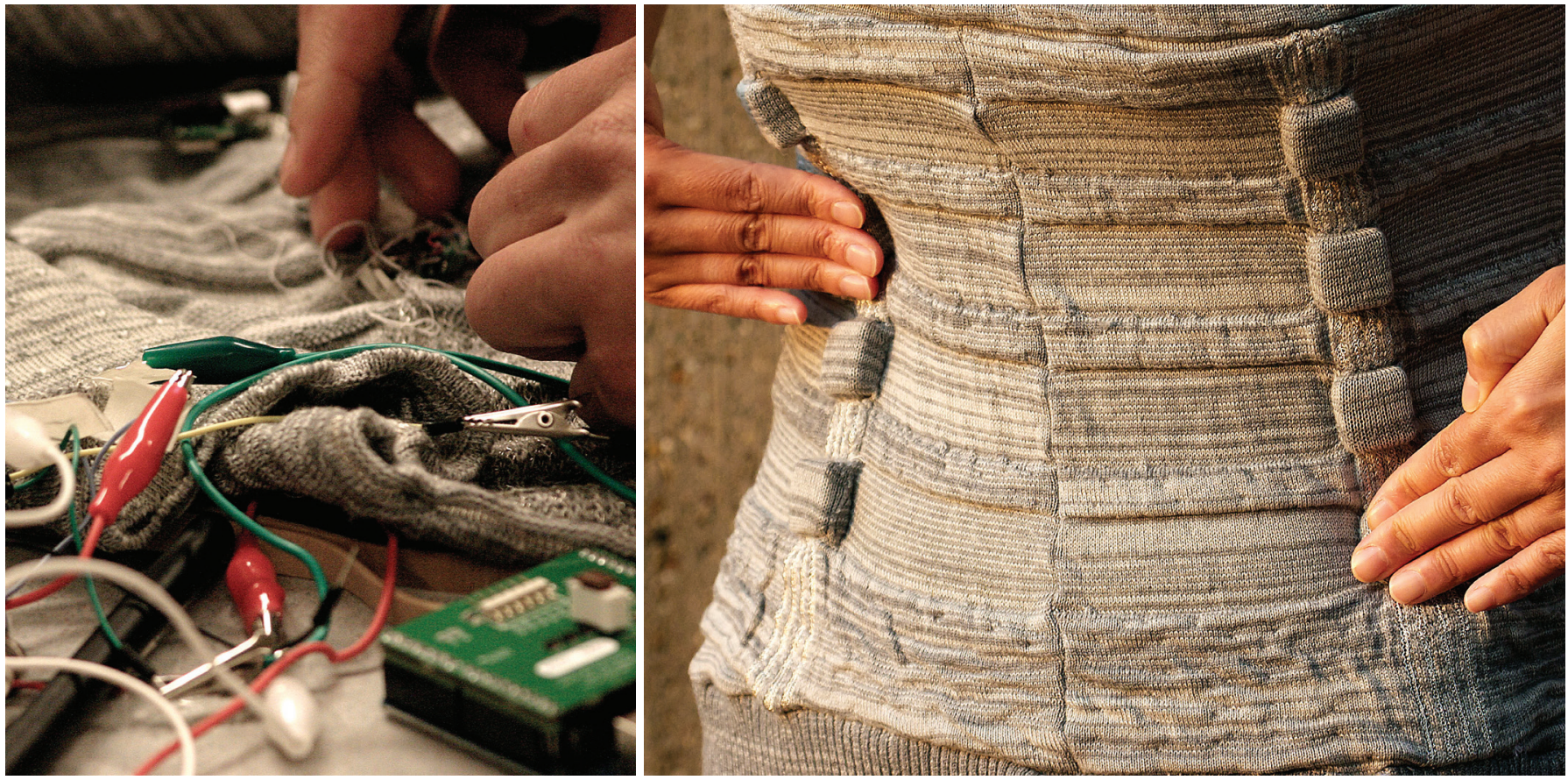

$\rightarrow$ Construction and finished detail on Vibe-ing, a merino wool garment that invites the body to feel, move, and heal through vibration therapy.

Moreover, WS has developed a strong network of industry partners (regional, national, and international) and in this way receives support on different levels from both the textile and the electronics world. The composition of the staff reflects our relation with industry. Industry professionals have an important role in coaching students and extending the network by involving clients from industry who can propose design briefs for students. For example, Marina Toeters, who combines her work as a fashion designer with student coaching at WS, developed the design brief Worn Identity, where students have to think about the societal impact of customizable and interactive fashion and design product-service systems for these opportunities. By having industry and other stakeholders involved in setting up these design briefs, we make sure the projects have a level of societal relevance. Finally, industry collaborates with WS on the realization of projects developed by students and researchers.

\section{Describe a day in the life of your lab.} The general rhythm and mood of the lab is vibrant. Depending on the phase of the semester, we have either curious, focused, or nervous students generating the vibe of the space.

A "typical" day in our lab is filled with lively, inspirational, often unexpected events and activities. The range of activities varies from cutting and pasting scientific submissions or knitted fabrics, to soldering and sewing haptic pillows, to sketching and prototyping bed sheets or storytelling services. Throughout the day, questions from others and visits from guests shift people's attention from current activities to new opportunities.

In a self-maintaining and open lab, everybody shares skills and knowledge, helps fix the machines, and introduces new materials and best practices. These material explorations, prototypes, and sketches are openly tried out and discussed to get collaboration going in a critical and constructive way. For example, the final results of a fashion shoot lead to a strategic discussion on how to visualize embodied wearables in research. Meanwhile, the fashion intern is discussing the details of a sewing pattern with a researcher, who wears the garment herself to optimize the position of the vibration motors in relation to the silhouette of the garment.
As the lab is also an inspirational test bed for the regional industry, we often welcome visitors and show them our prototypes to inspire them and inform them about the opportunities and challenges of integrating computing and smart textiles.

\section{What is one feature of your lab that} you could not do without? We are convinced that developing valuable wearables requires a hands-on approach that actively involves all stakeholders, including the intended user, from a very early stage. It was only through this approach, working on smart textiles in the context of wearables, that we became aware of the bottlenecks that we need to resolve in order to make progress. Actually, we found that creating an approach for the design of smart textiles is almost a design process in itself. In this process the whole context of the development of smart textiles for well-being is created and becomes alive, leading to a community of practitioners who help and challenge each other. From this process we gained a better awareness of the real challenges within this field. We are sure we have identified some necessary ingredients, such 

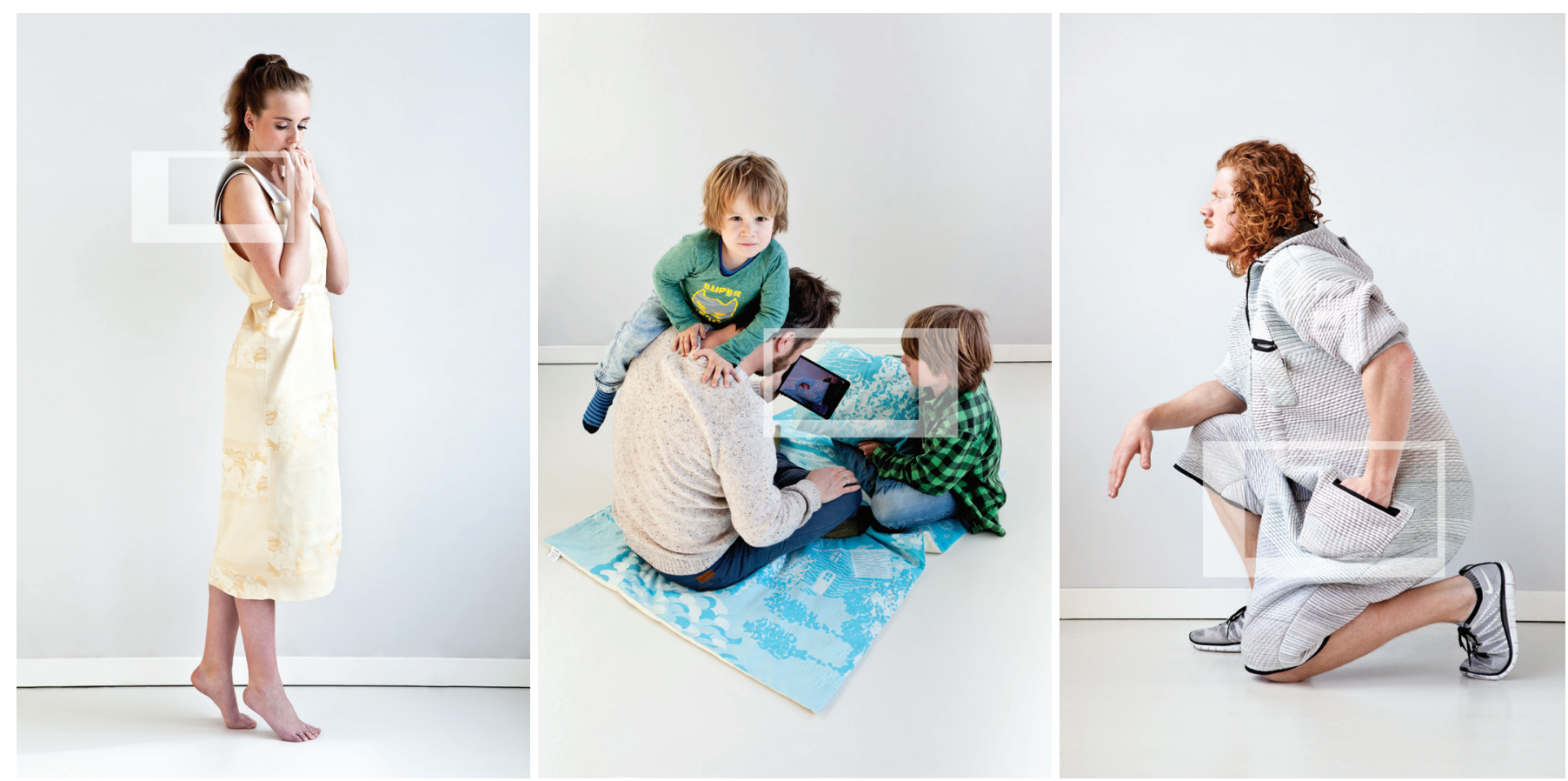

as appropriate ways of field testing, interacting, and bringing all the stakeholders together.

\section{What is one feature of your lab you want and do not have? Research on wearables can be done at the yarn level, the textile level, or the product/ garment level, and we believe that all of them are important. In Wearable Senses we work with existing off- the-shelf technologies, as one of our main research interests is how to bring technology closer to the body (research on a product/garment level). We are currently starting to develop our own electronic components; having dedicated technology supports us in going further in the textile integration in order to do research on a textile level. However, we are still working with hard PCB boards. The next step is to get into flexible and printed ones.}

\section{What is most challenging about the work you do here? From our experience with the Wearable Senses theme, it became increasingly clear that there are at least three}

challenges we need to tackle if we want to effectively explore the design opportunities related to well-being. First, it is more difficult than expected to move away from traditional screen- and desktop-based interaction metaphors. We need to develop completely new and different interaction styles, integrating bodily actions, cognition, and perception, and adopting theoretical frameworks that support embodied action and perception. Second, combining textiles and electronics to create high-quality and robust prototypes is technically challenging and requires new solutions and techniques that we cannot possibly develop in our own labs. Third, we find it is hard to predict the effect of our designs on daily life and that we need to develop effective approaches to measure and evaluate our concepts in the real-life context. The three challenges - interaction styles for close to the body, new collaboration networks, and pools of in-situ tested examples - gave direction to our research efforts and determined the emphasis of some of the research projects that were initiated.

\section{Creating an approach for the design of smart textiles is almost a design process in itself.}

